Revista Calidad en la Educación Superior Programa de Autoevaluación Académica Universidad Estatal a Distancia, Costa Rica ISSN 1659-4703

revistacalidad@uned.ac.cr

\title{
REFLEXIONES QUE PROMUEVEN LA CALIDAD EN LOS PROCESOS DE FORMACIÓN DOCENTE DE LA CARRERA DE EDUCACIÓN ESPECIAL CON ÉNFASIS EN INTEGRACIÓN DE LA UNIVERSIDAD NACIONAL
}

\author{
REFLECTIONS THAT PROMOTE QUALITY IN THE PROCESSES OF TEACHER \\ TRAINING OF THE SPECIAL EDUCATION WITH EMPHASIS IN INTEGRATION \\ CAREER OF THE UNIVERSIDAD NACIONAL
}

\begin{abstract}
Heidy León Arce ${ }^{1}$, heidy.leon.arce@una.cr Ana Lucía Chaves Álvarez², analuchavesalvarez@gmail.com Ana Isabel Herrera Castro ${ }^{3}$, ana.herrera.castro@una.cr Sylvia Segura Esquivel ${ }^{4}$, silvia.segura.esquivel@una.cr Ana María Hernández Segura ${ }^{5}$, ana.hernandez.segura@una.cr Lilliam Susana Ruiz Guevara6, susanaruizguevara@gmail.com Universidad Nacional, Costa Rica
\end{abstract}

La educación verdadera es praxis, reflexión y acción del hombre sobre el mundo para Volumen 6, número 1 transformarlo. Paulo Freire Mayo 2015

Pp. $213-248$

Recibido: mayo, 2014

Aprobado: 30 de abril, 2015

\footnotetext{
${ }^{1}$ Heidy León Arce. Educadora y académica universitaria, Máster en Pedagogía con Énfasis en Diversidad de los Procesos Educativos, Universidad Nacional. Licenciada en Educación Especial con Énfasis en Integración, Universidad Nacional, Costa Rica. Académica de la carrera de Educación Especial con Énfasis en Integración, Universidad Nacional, C.R..

${ }^{2}$ Ana Lucía Chaves Álvarez. Educadora, Máster en Pedagogía con Énfasis en Desarrollo y Atención de la Primera Infancia, Universidad Nacional, Costa Rica. Trabajó como académica de la carrera de Educación Preescolar, División de Educación Básica, y como investigadora del Instituto de Estudios Interdisciplinarios de la Niñez y la Adolescencia, CIDE, Universidad Nacional, C.R.

${ }^{3}$ Ana Isabel Herrera Castro. Educadora, Máster en Integración de Personas con Discapacidad, Universidad de Salamanca. Académica de la carrera de Educación Especial con Énfasis en Integración, Universidad Nacional, C.R.

${ }^{4}$ Sylvia Segura Esquivel. Educadora, Máster en Pedagogía con Énfasis en Diversidad en los Procesos Educativos. Académica e investigadora en la carrera de Pedagogía con Énfasis en I y II Ciclos de la EGB, División de Educación Básica-CIDE-UNA.

${ }^{5}$ Ana María Hernández Segura. Educadora, Doctora en Educación de la Universidad Estatal a Distancia (UNED), Máster en Educación con Mención en Docencia Universitaria, Universidad Nacional, Costa Rica. Profesora de la carrera de Educación Preescolar, División de Educación Básica-CIDE-UNA.

${ }^{6}$ Lilliam Susana Ruiz Guevara. Educadora e investigadora, Doctora en Educación de la Universidad Estatal a Distancia (UNED), Máster en Administración Educativa, Universidad La Salle, Licenciada en Educación Preescolar de la Universidad Nacional. Académica de la carrera de Educación Preescolar, División de Educación Básica-CIDE-UNA.
} 
Reflexiones que promueven la calidad en los procesos de formación docente de la carrera de Educación Especial con Énfasis en Integración de la Universidad Nacional

Heidy León Arce, Ana Lucía Chaves Álvarez, Ana Isabel Herrera Castro, Sylvia Segura Esquivel, Ana María Hernández Segura y Lilliam Susana Ruiz Guevara

\section{Resumen}

Este artículo expone reflexiones sobre algunos aspectos recopilados desde la perspectiva de la población estudiantil en el proceso de autoevaluación de la carrera de Educación Especial con Énfasis en Integración (CEEI), de la División de Educación Básica (DEB) del Centro de Investigación y Docencia en Educación (CIDE) de la Universidad Nacional (UNA), Costa Rica, ante el Sistema Nacional de Acreditación de Educación Superior (SINAES) en el año 2011.

Los insumos para la reflexión a lo interno del equipo de mejoramiento continuo se basan en la opinión de 118 estudiantes regulares de la CEEI, como actores del proceso de autoevaluación. El propósito que se cumple es lograr la identificación de aspectos para la mejora de la carrera. Por su parte, las opiniones y las reflexiones presentadas en el artículo giran en torno a las características deseables del estudiantado que ingresa a la CEEI, al vínculo de la carrera con el contexto y la realidad y a las investigaciones afines a temáticas de la CEEI.

Las principales conclusiones del análisis revelan que haber indagado sobre las características deseables del estudiantado va más allá de una priorización de estas. El desafío es pensar en la carrera no solo desde las características particulares del estudiante universitario, como su perfil o su condición, sino desde el desarrollo de acciones y estrategias que favorezcan los procesos de formación. Por otra parte, la línea de investigación-acción en la CEEI es vista como una respuesta a las necesidades socioeducativas, las cuales son visualizadas desde las prácticas pedagógicas de los cursos, o bien desde las propuestas de los trabajos finales de graduación. De ahí que se vislumbren nuevos espacios emergentes en el ámbito escolar y fuera de él. Además, apunta hacia una mirada metodológica más diversa, amplia y humana que es promovida por las interacciones, la convivencia y la proximidad con el contexto real y lo cotidiano.

Palabras claves: autoevaluación; estudiantes; características deseables; relación teoría-práctica; contexto; investigaciones

\section{Abstract}

This paper presents reflections on some topics collected from the perspective of the student population in the self-assessment process conducted at the Special Education Major with an Emphasis in Integration (CEEI), Basic Education Division (DEB), Center for Research and Teaching in Education (CIDE) at the National University (UNA) of Costa Rica, presented to the National Accreditation System for Higher Education (SINAES) in 2011.

The input used for internal reflection by the continuous quality improvement team is based on the opinion of 118 regular students of the CEEI, who were actors during the self-assessment process. The achieved goal was to identify factors which contributed to improving the program. 
Reflexiones que promueven la calidad en los procesos de formación docente de la carrera de Educación Especial con Énfasis en Integración de la Universidad Nacional

Heidy León Arce, Ana Lucía Chaves Álvarez, Ana Isabel Herrera Castro, Sylvia Segura Esquivel, Ana María Hernández Segura y Lilliam Susana Ruiz Guevara

The opinions and reflections presented in this article revolve around the desirable characteristics of students entering the CEEl; the program's bond with context and reality, as well as research related to the CEEl topics.

The main findings of the analysis reveal that the research of the desirable characteristics of students, goes beyond their prioritization. The challenge is to think of the program, not only from the particular characteristics of the student, such as their profile or condition, but also from the development of strategies and actions that favor the formation processes.

Moreover, the research-action line at CEEI is seen as a response to social and educational needs, which are visualized from the pedagogical practices of the courses, or from the graduation paper proposals. Hence, new emerging spaces in schools and beyond are perceived. It also requires a shift into a more human, diverse, comprehensive methodology, which is promoted by interactions, coexistence and proximity to the current context and the everyday scenario.

Keywords: Self-assessment, students, desirable characteristics, theory-practice relationship, context, research.

La DEB, como unidad académica desde hace más de tres décadas, es responsable de los procesos de formación docente en las carreras de educación, en este caso la CEEI. Asimismo, promueve la autoevaluación a partir del año 2000, asumiendo dichos procesos con miras a la acreditación de las carreras; de esta forma, la CEEI logró en el año 2002 la primera acreditación ante el SINAES y en el año 2007 y 2012 se revalidó una segunda y tercera acreditación.

Escuchar y tomar en cuenta las voces de los actores que forman parte de los procesos de formación universitaria, en este caso la población estudiantil, es fundamental para la institución, así se ha determinado desde la fundación de la UNA, en el año 1974, cuando uno de sus precursores, Benjamín Núñez, manifestó que "debe entenderse que el estudiante se constituye de hecho en arquitecto de todo lo que complemente su capacitación central básica, a partir de los intereses que despierta en él, el conocimiento científico de la realidad" (Núñez, 1974, citado por UNA, 2005, p. 8). Por tanto, es importante mirar al estudiante como un actor fundamental de todo proceso educativo y formativo desde el ideal de una 
Reflexiones que promueven la calidad en los procesos de formación docente de la carrera de Educación Especial con Énfasis en Integración de la Universidad Nacional

Heidy León Arce, Ana Lucía Chaves Álvarez, Ana Isabel Herrera Castro, Sylvia Segura Esquivel, Ana María Hernández Segura y Lilliam Susana Ruiz Guevara

universidad que responda a una meta de formación que contribuye a la transformación de las realidades socioeducativas.

Es así que en el 2005 el Modelo pedagógico de la UNA continua en la línea de promover en la comunidad estudiantil «una participación activa en la construcción de la vida universitaria y, especial en los procesos educativos» (p. 8).

A la luz de lo anterior, la voz del estudiantado participante en los procesos de autoevaluación permite identificar elementos para lograr una mayor pertinencia y significatividad en los procesos de formación docente. Estos, a su vez, promueven un aprovechamiento de las experiencias para entablar discusiones reflexivas sobre problemas reales que acontecen a lo interno de la CEEI (Freire, 2009).

Para ello, los y las estudiantes, mediante una encuesta, llenaron un cuestionario vía electrónica por medio de la plataforma Lime Survey, administrada por el Programa de Desarrollo y Gestión Curricular de la Universidad Nacional. Cabe señalar que los tres tópicos que se desarrollan y se proponen en el instrumento fueron diseñados y validados por el Programa de Desarrollo y Gestión Curricular de la UNA y el equipo de mejoramiento continuo de las carreras de la DEB, por lo tanto se planteó en la siguiente línea: las características deseables, las cuales se entienden como un cúmulo de habilidades y aptitudes que favorecen al estudiantado que aspira a ingresar a una carrera universitaria; las mismas se convierten en un reto para el proceso de formación docente que debe partir de dichas características y potenciarlas, o bien promover las mejoras para la permanencia del estudiantado. Por otra parte, se reconoce que dicho grupo de habilidades resulta una tarea compleja en su definición y determinación, pues las habilidades son cambiantes ante la incertidumbre que tiene la persona docente en tiempos en los que las competencias van más allá de las que se plantean en el presente escrito. Otra de las líneas de reflexión se argumenta con la realidad y la 
Reflexiones que promueven la calidad en los procesos de formación docente de la carrera de Educación Especial con Énfasis en Integración de la Universidad Nacional

Heidy León Arce, Ana Lucía Chaves Álvarez, Ana Isabel Herrera Castro, Sylvia Segura Esquivel, Ana María Hernández Segura y Lilliam Susana Ruiz Guevara

relación con los diversos contextos, y promueve la carrera como parte de su relación teoría-práctica y mediante el eje práctico pedagógico; esta mirada favorece el cambio y la transformación de las realidades socioeducativas, los apropia y logran comprender y dar significado al proceso de formación. Por último, la incorporación de los trabajos finales de graduación, tanto en ámbitos formales y no formales, abren a su vez espacios emergentes que contribuyen a la pertinencia del plan de estudios; por tanto, el componente de investigación emerge como un pilar fundamental en los procesos de formación universitaria y específicamente en los procesos de formación de la CEEI.

Puede considerarse entonces que este proceso de autoevaluación de la CEEI, en el que los y las estudiantes tienen un papel fundamental, es una posibilidad para ver el presente, visualizar el futuro y buscar opciones para mejorar.

\section{Contextualización del plan de estudios de la CEEI}

La carrera de Educación Especial de la UNA ha mantenido una reflexión continua a lo largo de los últimos veinticinco años con el propósito de desarrollar un plan de estudios pertinente, acorde con los planteamientos de las normativas internacionales, que dé respuesta a nivel nacional a las necesidades socioeducativas y forme profesionales con una visión humanista y de cambio. Lo anterior se ha enriquecido gracias a los procesos de autoevaluación con miras a la reacreditación ante el Sistema Nacional de Acreditación de la Educación Superior (SINAES) de la carrera y a los aportes de empleadores y empleadoras, personas graduadas, estudiantes y personal académico, que permiten una mirada espejo del quehacer académico en los procesos de formación en aspectos relacionados con la vinculación con la realidad, el fortalecimiento de una conciencia crítica, la capacidad de enfrentar el cambio y adaptarse a nuevas situaciones y el análisis reflexivo de su práctica pedagógica, entre otros aspectos. 
Reflexiones que promueven la calidad en los procesos de formación docente de la carrera de Educación Especial con Énfasis en Integración de la Universidad Nacional

Heidy León Arce, Ana Lucía Chaves Álvarez, Ana Isabel Herrera Castro, Sylvia Segura Esquivel, Ana María Hernández Segura y Lilliam Susana Ruiz Guevara

La toma de conciencia en las tendencias de formación sobre los modelos de apoyo educativo que se están desarrollando nos enfrenta a una transición entre dos de ellos, el caracterizado por enfatizar en el apoyo jerárquico y transmisor, y el otro por ser constructivo y colaborativo (Comisión de Expertos de Educación Especial, 2004). La posibilidad y necesidad de migrar de un patrón a otro constituye para el personal académico de la carrera un espacio para la reflexión, la clarificación y la operacionalización del plan de estudios. Transitar de una visión de formación que promueve un sentido de especialista que ofrece «la solución» a un profesional que pretende que las propuestas alternativas se construyan en conjunto, con aportes de diversas perspectivas en un marco de trabajo colaborativo y cooperativo, es un trabajo que requiere de una intencionalidad explícita del colectivo de académicos y académicas responsables de la formación docente para vislumbrar rutas que contribuyan a fortalecer dichas habilidades y capacidades en los futuros docentes. Pues no solo basta con que el plan de estudios se enmarque dentro de la propuesta de un modelo de apoyo constructivo o colaborativo, sino que requiere estrategias concretas que se plasmen en las prácticas pedagógicas de los y las estudiantes.

La estructura del plan de estudios contempla, por un lado, el eje práctico pedagógico que se constituye en una práctica continua y sistemática a lo largo de la carrera, de este modo, posibilita una interacción permanente con el medio socioeducativo y laboral. Y, por otro lado, el área científico-pedagógica constituida «por aquellos conocimientos teóricos, conceptuales y metodológicos, básicos e imprescindibles en la labor docente de los futuros graduados. Esta área se sustenta en la complejidad del fenómeno educativo» (UNA, 2008, p. 60), para lo cual articula dos componentes: el de la praxis pedagógica y el de la mediación pedagógica. 
Reflexiones que promueven la calidad en los procesos de formación docente de la carrera de Educación Especial con Énfasis en Integración de la Universidad Nacional

Heidy León Arce, Ana Lucía Chaves Álvarez, Ana Isabel Herrera Castro, Sylvia Segura Esquivel, Ana María Hernández Segura y Lilliam Susana Ruiz Guevara

El concepto de praxis se entiende como un proceso de acción y de reflexión en un diálogo permanente del estudiantado con la realidad socioeducativa, lo cual genera una mayor conciencia y pertinencia de las respuestas que deberán aportar posteriormente como profesionales.

Asimismo, el concepto de mediación pedagógica comprende la coherencia entre la función de un docente y la capacidad de construcción y significatividad en los procesos de aprendizaje, y le ofrece al estudiantado un ambiente potencializador a partir de sus interpretaciones de forma colectiva e individual, para reinterpretar el mundo y sus realidades.

Es así como el Plan de estudios de la carrera de Educación Especial con Énfasis en Integración considera la complejidad del fenómeno educativo en la medida en la que "se desarrolla la construcción de un profesional reflexivo, investigador e innovador, con capacidad de desarrollar propuestas pedagógicas que potencien y garanticen la igualdad de oportunidades con equidad en el acceso a la educación en el marco de los derechos humanos» (UNA, 2008, p. 60).

Se pretende, por lo tanto, una relación dialéctica entre teoría y práctica:

Y con ello la posibilidad de que cada actor del proceso pueda construir aprendizajes más significativos y duraderos que emerjan de la confrontación, del análisis y reflexión de la realidad situacional en la que está inmerso y de la valoración de los alcances de la teoría que fundamenta sus experiencias (UNA, 2008, p. 47).

De esta manera, los últimos planteamientos que se han llevado a cabo para el diseño y la ejecución del plan de estudios, que aún se encuentra en proceso de reflexión y análisis, consideran que la intervención pedagógica juega una función primordial en la formación docente. Para esto se propone una estrategia 
Reflexiones que promueven la calidad en los procesos de formación docente de la carrera de Educación Especial con Énfasis en Integración de la Universidad Nacional

Heidy León Arce, Ana Lucía Chaves Álvarez, Ana Isabel Herrera Castro, Sylvia Segura Esquivel, Ana María Hernández Segura y Lilliam Susana Ruiz Guevara

metodológica y un eje longitudinal y transversal en la estructura curricular del plan de estudios, organizado en torno a cursos de praxis como un eje transversal metodológico que integra la investigación, la reflexión y la identidad profesional.

Así, la formación docente se basa en aspectos tales como el cambio, la transformación, la relación activa entre los diferentes actores de los procesos educativos, la atención a la diversidad y las diferentes realidades sociales y de contextos; todo esto con el fin de propiciar una educación con sentido y significado.

A continuación se explicita un marco referencial breve desde donde se consideran los aspectos a analizar en este artículo, con el propósito de que el lector y la lectora tengan claridad sobre nuestra postura en el marco del mejoramiento continuo de la CEEI, derivado de los procesos de autoevaluación y acreditación, y mediante el cual se develan todas aquellas fortalezas y debilidades del quehacer de la carrera y de esta manera se identifican todos los elementos indispensables para mantenerla actualizada y brindarle al estudiantado una oferta educativa de acuerdo a las demandas de la sociedad.

\section{Características deseables en los y las estudiantes que ingresan a la CEEI}

Por características deseables se entiende un conjunto de habilidades y aptitudes ideales o favorables que debería tener el o la estudiante que aspira a ingresar a la carrera y que le permitirán iniciar el proceso de formación universitaria. En este sentido, el proceso de formación docente debería partir de dichas características para potenciarlas, así como desarrollar los saberes conceptuales, procedimentales y actitudinales establecidos en el plan de estudios.

Tedesco y Tenti Fanfani (2002) argumentaron que «si alguien quisiera hacer un listado de lo que se consideran las "características deseables" del "nuevo docente" 
Reflexiones que promueven la calidad en los procesos de formación docente de la carrera de Educación Especial con Énfasis en Integración de la Universidad Nacional

Heidy León Arce, Ana Lucía Chaves Álvarez, Ana Isabel Herrera Castro, Sylvia Segura Esquivel, Ana María Hernández Segura y Lilliam Susana Ruiz Guevara

a partir de una revisión de la literatura contemporánea, seguramente encontraría una serie de elementos muy variados y hasta contradictorios» (p.4). De igual manera, resulta una tarea compleja definir las características deseables de un o una estudiante para el ingreso a un nivel de educación superior.

Partiendo del argumento anterior, en el que se evidencia que se hace difícil definir un conjunto determinado de características únicas y previas para el proceso de formación docente, se considera pertinente explorar, a partir de las opiniones de los y las estudiantes, sobre este aspecto. Es así que se le consultó a un grupo de estudiantes de la CEEl, en el proceso de autoevaluación con miras a la reacreditación ante el Sistema Nacional de Acreditación de la Educación Superior (SINAES), sobre algunas posibles características deseables dadas para indagar su opinión, con el propósito de ir configurando un marco referencial con el que la carrera pueda contar para fortalecer y mejorar los procesos formativos. Las características que se seleccionaron fueron: sensibilidad para trabajar con personas con discapacidad, creatividad y dinamismo, fluidez verbal y escrita, habilidad para enseñar y destrezas interpersonales, entre otras.

Al respecto, Bertoni mencionó que es pertinente

Conjugar la demanda de competencias deseables en las y los estudiantes, características que deberían poseer y no poseen, con las condiciones académicas emergentes de las diversas investigaciones que dan cuenta de la situación real con que los bachilleres abordan los estudios universitarios. La conjunción de estas informaciones proporcionarán los insumos necesarios para visualizar la distancia que media entre un perfil de ingreso lo más próximo a la realidad y el nivel formativo reclamado por la Universidad (2005, p. 2). 
Reflexiones que promueven la calidad en los procesos de formación docente de la carrera de Educación Especial con Énfasis en Integración de la Universidad Nacional

Heidy León Arce, Ana Lucía Chaves Álvarez, Ana Isabel Herrera Castro, Sylvia Segura Esquivel, Ana María Hernández Segura y Lilliam Susana Ruiz Guevara

En este sentido, se construye un apartado del cuestionario aplicado a los y las estudiantes de la CEEI en el proceso de autoevaluación, destinado a explorar las características mencionadas que a continuación se detallan.

La sensibilidad para trabajar con personas con discapacidad es una de ellas, lo que implica tener tacto, y este lo circunscribe dentro del marco de las relaciones humanas y, específicamente, en las relaciones que establece la persona docente para desarrollar una pedagogía de la diversidad. Zúñiga destacó lo siguiente:

El tacto o sensibilidad tiene propiedades interpersonales y normativas que parecen especialmente adecuadas para las interacciones pedagógicas con los niños.

El tacto pedagógico se manifiesta principalmente como una orientación consciente en cuanto a la forma de ser y actuar con los niños. No se trata de la manifestación de algunos comportamientos observables como de una posición activa en las relaciones $(2010$, p. 3$)$.

Se puede afirmar entonces que para poder desarrollarse como docente de Educación Especial y, por ende, potenciar en su proceso formativo la sensibilidad es una característica que le permitirá «responder a los principios de comprensividad y de atención a la diversidad» (Zúñiga, 2010, p. 1) y, por supuesto, a las particularidades de todas las personas con las que interactúe.

Otra de las características considerada es la creatividad, concepto, como muchos otros, difícil de definir, sin embargo, la propuesta de Bruner es interesante, ya que parte de que creamos cuando "relacionamos de distinta forma elementos ya existentes, pues las formas de creatividad surgen de una nueva actividad combinatoria" ([1978], Bruner citado por Martínez-Salanova, 2002, p. 11.). 
Reflexiones que promueven la calidad en los procesos de formación docente de la carrera de Educación Especial con Énfasis en Integración de la Universidad Nacional

Heidy León Arce, Ana Lucía Chaves Álvarez, Ana Isabel Herrera Castro, Sylvia Segura Esquivel, Ana María Hernández Segura y Lilliam Susana Ruiz Guevara

Para la carrera es importante vincular la creatividad y a su vez el dinamismo como elementos existentes, ya que permite hablar de espacios e intenciones que fomenten la interacción entre elementos y sujetos, debido a que ambos aspectos son centrales en los procesos de enseñanza y aprendizaje. El concepto de dinamismo se entiende como una acción vital producto de interacciones que posibilita movilizaciones de toda índole en el desarrollo de procesos educativos.

Por otra parte, la fluidez verbal y escrita es otra de las características elegidas para consultarles a los y las estudiantes, con el propósito de conocer si es considerada una competencia básica y fundamental en las interacciones pedagógicas. Algunos estudios mencionan la lengua como una característica deseable, tal como lo expresaron Lima, Kahan, García, Ríos, Cha y Plachot. (2004):

Los estudiantes (...) deben haber adquirido competencias lingüísticas, manejando amplio vocabulario y fluidez en la expresión oral y escrita. En el lenguaje escrito, se requiere el manejo de las distintas complejidades escriturales (texto narrativo, expositivo y argumentativo), así como un adecuado uso de la sintaxis, del léxico cotidiano y académico y de la ortografía (p. 1).

De esta manera, conocer la opinión de los y las estudiantes sobre este aspecto permite contemplarlo dentro del posible abanico de alternativas que la carrera considera para dar seguimiento a los procesos de formación docente.

La disposición hacia la enseñanza, entendida como una actitud positiva para enseñar, es otro elemento sobre el que se indagó en aquellos estudiantes que decidieron estudiar Educación Especial. En este sentido, y como respuesta a una educación que trasciende la trasmisión de conocimientos, la elaboración de respuestas «correctas», o como lo planteó Freire: la educación bancaria, se trata 
Reflexiones que promueven la calidad en los procesos de formación docente de la carrera de Educación Especial con Énfasis en Integración de la Universidad Nacional

Heidy León Arce, Ana Lucía Chaves Álvarez, Ana Isabel Herrera Castro, Sylvia Segura Esquivel, Ana María Hernández Segura y Lilliam Susana Ruiz Guevara

de tomar como base esta disposición, con el fin de que le permita a cada individuo desarrollar todo su potencial y que favorezca la posibilidad de preguntar, de construir aprendizajes de acuerdo a sus características. En suma, se trata de formar a un o una docente que, más que dar respuestas, tenga la habilidad de enseñar a aprender (Sartori y Castilla, 2004). Esta habilidad para enseñar supone tener entrega, compromiso por lo que se hace, conocer la realidad en la que se trabaja, asumir retos, construir estrategias que respondan a las particularidades de las personas y de los contextos en los que se desenvuelven.

Las relaciones interpersonales se encuentran entre las características deseables por la implicación que tienen en los procesos de enseñanza y aprendizaje. Para algunos autores dichas habilidades dependen del carácter de la persona, y por tanto no se pueden enseñar ni aprender (Lucha y Baerlocher, 2008). Se considera la segunda opción como pertinente en los procesos de formación docente. Para Pereira (2010), «las relaciones interpersonales en el ámbito humano cobran vital sentido desde el nacimiento y se van conformando en los procesos de interacción y socialización. El proceso educativo no está exento de procesos comunicativos e interactivos» (p.1).

Precisamente de los planteamientos del autor se infiere que las destrezas interpersonales son necesarias para trabajar en la diversidad, para desarrollar estrategias de trabajo en equipo, para ser tolerante, empático y respetuoso con las demás personas. Asimismo, los y las estudiantes participantes del proceso de acreditación de la CEEI consideraron algunas características deseables, como tener capacidad de innovar en la práctica, la creatividad y el dinamismo, elementos que deben ser inherentes a la función docente. Se puede afirmar entonces que todos estos aspectos constituyen características que deben poseer los y las docentes de educación especial y, por ende, las personas que deciden 
Reflexiones que promueven la calidad en los procesos de formación docente de la carrera de Educación Especial con Énfasis en Integración de la Universidad Nacional

Heidy León Arce, Ana Lucía Chaves Álvarez, Ana Isabel Herrera Castro, Sylvia Segura Esquivel, Ana María Hernández Segura y Lilliam Susana Ruiz Guevara

estudiar esta carrera, ya que en el ejercicio de su profesión deberán desarrollarlas al máximo.

\section{Realidad y relación de la CEEI con el contexto}

Ordoñez (2010) planteó que para Paulo Freire la educación implica comprender la situación en la que se vive, muy unido a transformar y actuar. De este argumento se deduce que para poder realizar un proceso de cambio y transformación en la realidad y para poder comprender las situaciones en las que se vive es necesario que en la formación docente se establezca una relación estrecha con los diversos contextos en los cuales se van a desenvolver los futuros profesionales, de manera que los conozcan, los hagan suyos, se identifiquen con ellos y así puedan encontrar sentido y significado a la formación universitaria que reciben. Todo lo anterior alude a una vinculación muy estrecha entre la teoría y la práctica, es decir a una relación dialéctica.

Es por esto que para la CEEI la relación con el contexto es un aspecto fundamental en el proceso de formación, y así lo reconocieron los y las estudiantes participantes en el proceso de autoevaluación cuando expresaron la vinculación desde los niveles iniciales de la carrera con el contexto educativo.

Cabe destacar que se propicia en la CEEl la relación con el contexto a través del eje práctico pedagógico, que por medio de los diferentes cursos de naturaleza teórico-práctica les permite a los y las estudiantes conocer e interactuar en espacios educativos desde el inicio de la carrera.

Asimismo, la carrera propone como culminación un periodo de intervención pedagógica, cada dos años del continuum del plan, entendido como un proceso que: 
Reflexiones que promueven la calidad en los procesos de formación docente de la carrera de Educación Especial con Énfasis en Integración de la Universidad Nacional

Heidy León Arce, Ana Lucía Chaves Álvarez, Ana Isabel Herrera Castro, Sylvia Segura Esquivel, Ana María Hernández Segura y Lilliam Susana Ruiz Guevara

Permite al estudiante-maestro, el llevar a la práctica escolar los conocimientos adquiridos en la Universidad, para construir verdaderos aprendizajes, fruto de la confrontación y sistematización de la práctica cotidiana del aula. Con estas experiencias los estudiantes deben retornar a las aulas universitarias con verdaderos insumos para la realimentación, la crítica y el debate de ideas (UNA, 2008, p.32).

Otras estrategias pedagógicas vinculadas a la relación con el contexto son las giras de campo, las visitas a diversas instituciones, la socialización de proyectos de investigación y los encuentros y las visitas de profesionales y expertos en los cursos, entre otras, que le permiten a la población estudiantil un acercamiento a la realidad y son el punto de partida para el debate crítico y el diálogo reflexivo de los y las estudiantes en el aula universitaria, lo cual promueve en los futuros docentes un análisis de su propia práctica, un conocimiento de los contextos en los cuales se desenvolverán, así como propuestas puntuales de intervención pedagógica que favorecen habilidades y actitudes para la transformación de dinámicas socioeducativas.

\section{Pertinencia del plan de estudios de la CEEl: una mirada a los temas de los trabajos finales de graduación}

De acuerdo con Tünnermann (2009), la pertinencia en la educación superior es un tema actual de debate. Al respecto, el autor señaló que en la Conferencia Regional Mundial quedó claro que "la obligación, tanto del sector público como del privado, es ofrecer una educación superior con calidad y pertinencia" (p.1).

Para el autor la pertinencia es un concepto amplio que no se reduce a las demandas del sector económico, por tanto planteó que «el concepto de pertinencia se ciñe así al papel que la educación superior desempeña en la 
Reflexiones que promueven la calidad en los procesos de formación docente de la carrera de Educación Especial con Énfasis en Integración de la Universidad Nacional

Heidy León Arce, Ana Lucía Chaves Álvarez, Ana Isabel Herrera Castro, Sylvia Segura Esquivel, Ana María Hernández Segura y Lilliam Susana Ruiz Guevara

sociedad y lo que esta espera de aquella [en negrita en el original] (Tünnermann, 2009, p.1).

La pertinencia tiene que ver con la misión y la visión de las instituciones de educación superior, es decir, con su ser y su deber ser, con la médula de su cometido, y no puede desligarse de los grandes objetivos y las necesidades de la sociedad en las que dichas instituciones están inmersas ni de los retos del nuevo contexto mundial. Por lo tanto, la pertinencia de la educación superior no se agota en su dimensión económica, sino que abarca sus otras dimensiones: laboral, social, cultural y ecológica (Tünnermann, 2009).

Ahora bien, es importante señalar que para efectos de este artículo se hace referencia a la pertinencia del plan de estudios de la CEEI en términos de los procesos de investigación, en la dimensión de los trabajos finales de graduación que llevan a cabo los y las estudiantes del nivel de licenciatura, en la medida en la que favorece el desarrollo profesional de las personas graduadas y el aporte al mejoramiento del sistema educativo, de las prácticas pedagógicas, de las comunidades, poblaciones vulnerables, etc. Al respecto, la Declaración Mundial sobre la Educación Superior en el Siglo XXI (1998), citada por Tünnermann (2009), destaca en relación con la pertinencia que «la educación superior debe aumentar su contribución al desarrollo del conjunto del sistema educativo, sobre todo mejorando la formación del personal docente, la elaboración de los planes de estudio y la investigación sobre la educación» (p.8).

Precisamente, se puede argumentar que propiciar la investigación en el ámbito educativo tiene que ver con la pertinencia, ya que permite producir «conocimiento sistematizado y valioso» (Sverdikc, Anderson, Augustowsky, Herr, Rivas y Suárez, 2007, p.19). Tal como lo plantearon los autores citados, el componente de investigación resulta fundamental para producir nuevo conocimiento, y en el 
Reflexiones que promueven la calidad en los procesos de formación docente de la carrera de Educación Especial con Énfasis en Integración de la Universidad Nacional

Heidy León Arce, Ana Lucía Chaves Álvarez, Ana Isabel Herrera Castro, Sylvia Segura Esquivel, Ana María Hernández Segura y Lilliam Susana Ruiz Guevara

ámbito de la educación este se puede considerar como una función primordial de los y las docentes, ya que es lo que les permitirá conocer sus realidades y transformarlas.

La Declaración Mundial de la UNESCO sobre la Educación Superior en el Siglo XXI (1998), citada en el Modelo Pedagógico de la Universidad Nacional (2005), argumenta dentro de la misión y las funciones de la educación superior lo siguiente: "La promoción, generación y difusión de conocimientos por medio de la investigación científica y tecnológica, la docencia, la extensión y la producción" (s. p.). Es por esto que el componente de investigación emerge como un pilar fundamental en los procesos de formación universitaria y específicamente en los procesos de formación de la CEEl.

\section{Metodología}

Los procesos de autoevaluación en los que participan las carreras de la División de Educación Básica son muy valiosos para la reflexión de su quehacer y en particular para la CEEl al impactar en la calidad de la formación docente. Todos estos procesos son de gran pertinencia al contar con la participación de diferentes actores, entre ellos estudiantes, personas egresadas, población académica, empleadores y empleadoras y personal administrativo.

Como parte del proceso de autoevaluación, el SINAES les ofrece a las diferentes instituciones de educación superior un modelo a partir del cual estas deben guiarse para realizar sus procesos de autoevaluación. Este modelo se basa en criterios, indicadores, evidencias y estándares relacionados con todos los elementos con los cuales deben cumplir las carreras que se someten a dichos procesos de acreditación. 
Reflexiones que promueven la calidad en los procesos de formación docente de la carrera de Educación Especial con Énfasis en Integración de la Universidad Nacional

Heidy León Arce, Ana Lucía Chaves Álvarez, Ana Isabel Herrera Castro, Sylvia Segura Esquivel, Ana María Hernández Segura y Lilliam Susana Ruiz Guevara

Se considera importante anotar que los datos que se muestran en este artículo son producto de la participación directa de las personas egresadas de CEEI en el proceso de autoevaluación con miras a la reacreditación ante el Sistema Nacional de Acreditación de la Educación Superior (SINAES).

A partir de la identificación de los diferentes criterios establecidos por el SINAES se diseñan y aplican diferentes instrumentos, los cuales fueron elaborados y validados por el Programa de Desarrollo y Gestión Curricular de la UNA y el equipo de mejoramiento continuo de las carreras de la División de Educación Básica del Centro de Investigación y Docencia en Educación (CIDE) de la Universidad Nacional.

Como se mencionó anteriormente, estos instrumentos son dirigidos a los actores participantes: académicos, administrativos, egresados, empleadores y estudiantes, siendo estos últimos la base de la información obtenida.

Para efectos de este artículo se retoma la participación de los y las estudiantes de la CEEl. Se trabajó con un total de 118 estudiantes de 126 que tenía la CEEI, al momento del proceso de autoevaluación. El cuestionario se aplicó vía electrónica, es decir, cada estudiante completó el instrumento por medio de la plataforma Lime Survey, administrada por el Programa de Desarrollo y Gestión Curricular de la Universidad Nacional (UNA).

Cabe señalar que el cuestionario aplicado a los y las estudiantes se elaboró con setenta y dos preguntas distribuidas en seis secciones; sin embargo, para la presente reflexión se toma como referencia la segunda parte: Habilidades y Actitudes del Estudiantado y la sexta parte: Comentarios en los que la población estudiantil dio a conocer sus necesidades particulares y opiniones generales de la CEEI. 
Reflexiones que promueven la calidad en los procesos de formación docente de la carrera de Educación Especial con Énfasis en Integración de la Universidad Nacional

Heidy León Arce, Ana Lucía Chaves Álvarez, Ana Isabel Herrera Castro, Sylvia Segura Esquivel, Ana María Hernández Segura y Lilliam Susana Ruiz Guevara

Además, en el instrumento se utilizan escalas dicotómicas y diferentes variedades de escalas Likert que responden a las particularidades de los ítems, específicamente se trabaja con las escalas de muy de acuerdo, de acuerdo, parcialmente de acuerdo, en desacuerdo, muy en desacuerdo y siempre, casi siempre, a veces y nunca.

Posteriormente, el equipo de mejoramiento asumió el compromiso de reflexionar sobre los resultados que versan en cuanto a habilidades y experiencias de la carrera. De esta forma se trabajan los ítems y se dividen en tres grupos, a saber: características deseables, relación con el contexto y trabajos de investigación afines a la CEEI. Los argumentos son analizados para repensarlos desde la teoría y proponer a futuro consideraciones que la carrera debe tomar en cuenta para las mejoras respectivas.

\section{Características deseables en las y los estudiantes que ingresan a la CEEI}

El Plan de estudios de la CEEI (2008) incluye en su fundamentación teórica elementos indispensables que presentan las competencias del perfil del graduado. Dichas competencias se presentan por saberes conceptuales, procedimentales y actitudinales que deben ser operacionalizados en el proceso de formación de los y las estudiantes de la CEEI. No existe un perfil de ingreso a la carrera en el que se especifiquen las características deseables, por esa razón se les consultó a los y las estudiantes.

Como hemos comentado, conocer las características deseables se convierte en un insumo para enriquecer los procesos de formación desde la perspectiva del desarrollo de habilidades y actitudes en coherencia con el perfil de la persona graduada del plan de estudios 
Reflexiones que promueven la calidad en los procesos de formación docente de la carrera de Educación Especial con Énfasis en Integración de la Universidad Nacional

Heidy León Arce, Ana Lucía Chaves Álvarez, Ana Isabel Herrera Castro, Sylvia Segura Esquivel, Ana María Hernández Segura y Lilliam Susana Ruiz Guevara

En el marco del proceso de autoevaluación, al ofrecerles a los y las estudiantes regulares un listado sobre cuáles son las características deseables que debería poseer la población estudiantil que ingresa a la carrera sus respuestas se indican en la tabla 1, mostrándose las características en un orden de mayor a menor porcentaje, según las respuestas dadas en la escala muy de acuerdo.

Un $91,53 \%$ de la población estudiantil manifiesta estar muy de acuerdo con la característica deseable "sensibilidad para trabajar con personas con discapacidad"; este aspecto es valorado por los y las participantes y permite inferir que dicha característica puede ser un potenciador para la adquisición de mayor conciencia hacia las personas con condición de discapacidad. Pareciera que lo que mencionó Zúñiga (2010) sobre el tacto y la sensibilidad puede tener un peso importante en los procesos de formación docente en Educación Especial. Considerar las habilidades interpersonales, las interacciones, la actitud positiva y la comprensibilidad hacia la población con discapacidad como, según lo dicho por el autor, orientaciones conscientes que favorecen los procesos de atención con poblaciones vulnerables. No en vano es la característica deseable con un mayor porcentaje dentro de la población estudiantil consultada.

La segunda característica deseable es la "habilidad para enseñar" con un 86,44\% del total de respuestas de muy de acuerdo. De este modo se deduce que la disposición para enseñar podría estarse identificando como un elemento que favorece el proceso de formación docente a futuro, entendida como una actitud hacia la comprensión de la postura del otro, a ajustarse a las necesidades, responder a las particularidades, entre otras. 
Reflexiones que promueven la calidad en los procesos de formación docente de la carrera de Educación Especial con Énfasis en Integración de la Universidad Nacional

Heidy León Arce, Ana Lucía Chaves Álvarez, Ana Isabel Herrera Castro, Sylvia Segura Esquivel, Ana María Hernández Segura y Lilliam Susana Ruiz Guevara

Tabla 1. Porcentajes de opinión de las y los estudiantes de la CEEI sobre características deseables para ingresar a la carrera

\begin{tabular}{lr}
\hline Características deseables & Porcentaje \\
\hline Sensibilidad para trabajar con personas con discapacidad & \\
\hline En desacuerdo & $1,69 \%$ \\
\hline Parcialmente de acuerdo & $0,85 \%$ \\
\hline De acuerdo & $5,93 \%$ \\
\hline Muy de acuerdo & $91,53 \%$ \\
\hline & $100,00 \%$ \\
\hline Habilidad para enseñar & Total \\
\hline Muy en desacuerdo & \\
\hline Parcialmente de acuerdo & $0,85 \%$ \\
\hline De acuerdo & $0,85 \%$ \\
\hline Muy de acuerdo & $11,86 \%$ \\
\hline & $86,44 \%$ \\
\hline
\end{tabular}

Habilidades interpersonales

Muy en desacuerdo $\quad 0,85 \%$

Parcialmente de acuerdo $\quad 2,54 \%$

De acuerdo $11,86 \%$

Muy de acuerdo $84,75 \%$

Total $\quad 100,00 \%$

Creatividad y dinamismo

Muy en desacuerdo $\quad 0,85 \%$

$\begin{array}{ll}\text { Parcialmente de acuerdo } & 5,93 \%\end{array}$ 
Reflexiones que promueven la calidad en los procesos de formación docente de la carrera de Educación Especial con Énfasis en Integración de la Universidad Nacional

Heidy León Arce, Ana Lucía Chaves Álvarez, Ana Isabel Herrera Castro, Sylvia Segura Esquivel, Ana María Hernández Segura y Lilliam Susana Ruiz Guevara

\begin{tabular}{lr}
\hline De acuerdo & $14,41 \%$ \\
\hline Muy de acuerdo & $78,81 \%$ \\
\hline Fluidez verbal y escrita & Total \\
\hline Muy en desacuerdo & $100,00 \%$ \\
\hline En desacuerdo & $1,69 \%$ \\
\hline Parcialmente de acuerdo & $1,69 \%$ \\
\hline De acuerdo & $4,24 \%$ \\
\hline Muy de acuerdo & $38,14 \%$ \\
\hline & $54,24 \%$ \\
\hline
\end{tabular}

Fuente: Informe estudiantes regulares del proceso de autoevaluación de la CEEI 2010.

Chaves, Hernández, León, Ruiz y Segura (2010). Informe Paralelo: Cuestionario aplicado a Estudiantes Regulares de la carrera de Educación Especial. Proceso de Autoevaluación para la segunda Reacreditación. División de Educación Básica. Universidad Nacional (documento impreso, no publicado)

En tercer lugar, con un $84,75 \%$, se ubican las «habilidades interpersonales», característica que refleja la importancia que los y las estudiantes regulares le dan a las capacidades humanas que requieren para poder desenvolverse en esta carrera. Para Pereira (2010) «es indiscutible la función de las relaciones humanas en el ámbito educativo, en la conformación de un aprendizaje que no se circunscribe exclusivamente al aspecto cognitivo, sino que abarca la integralidad del ser humano» (p.11). Considerar esta característica nos acerca a propiciar espacios educativos interactivos favorables para los aprendizajes integrales.

Al mismo tiempo, el 78,81\% de los y las estudiantes están muy de acuerdo en que la creatividad y el dinamismo son necesarios dentro de las características de ingreso a la CEEI. Sin duda alguna, poseer dichas características incidirá de forma directa en el logro de la trascendencia en su función y en el entendimiento de "la 
Reflexiones que promueven la calidad en los procesos de formación docente de la carrera de Educación Especial con Énfasis en Integración de la Universidad Nacional

Heidy León Arce, Ana Lucía Chaves Álvarez, Ana Isabel Herrera Castro, Sylvia Segura Esquivel, Ana María Hernández Segura y Lilliam Susana Ruiz Guevara

labor pedagógica como un gesto de creatividad" (Toro, 2005, p. 45), en tanto se pueda buscar diferentes respuestas, traspasar los manuales que dicen qué hacer y cómo hacerlo y "pasar de la aplicación de lo que uno sabe a un aprender de aquello que aplica" (Toro, 2005, p.45).

Por último, el $54,24 \%$ de los y las estudiantes manifiestan estar muy de acuerdo en que una característica que debe tener la persona que quisiera ingresar a la CEEl es tener habilidades verbales y escritas que le permitan comunicarse de forma segura, acertada y fluida; esta es la característica con menor porcentaje, lo que revela la poca importancia que le da la población estudiantil.

Partiendo de lo anterior, explorar las características deseables proporciona los insumos para visualizar la distancia que media entre ellas y el nivel formativo que se pretende desarrollar en la carrera (Bertoni, 2005).

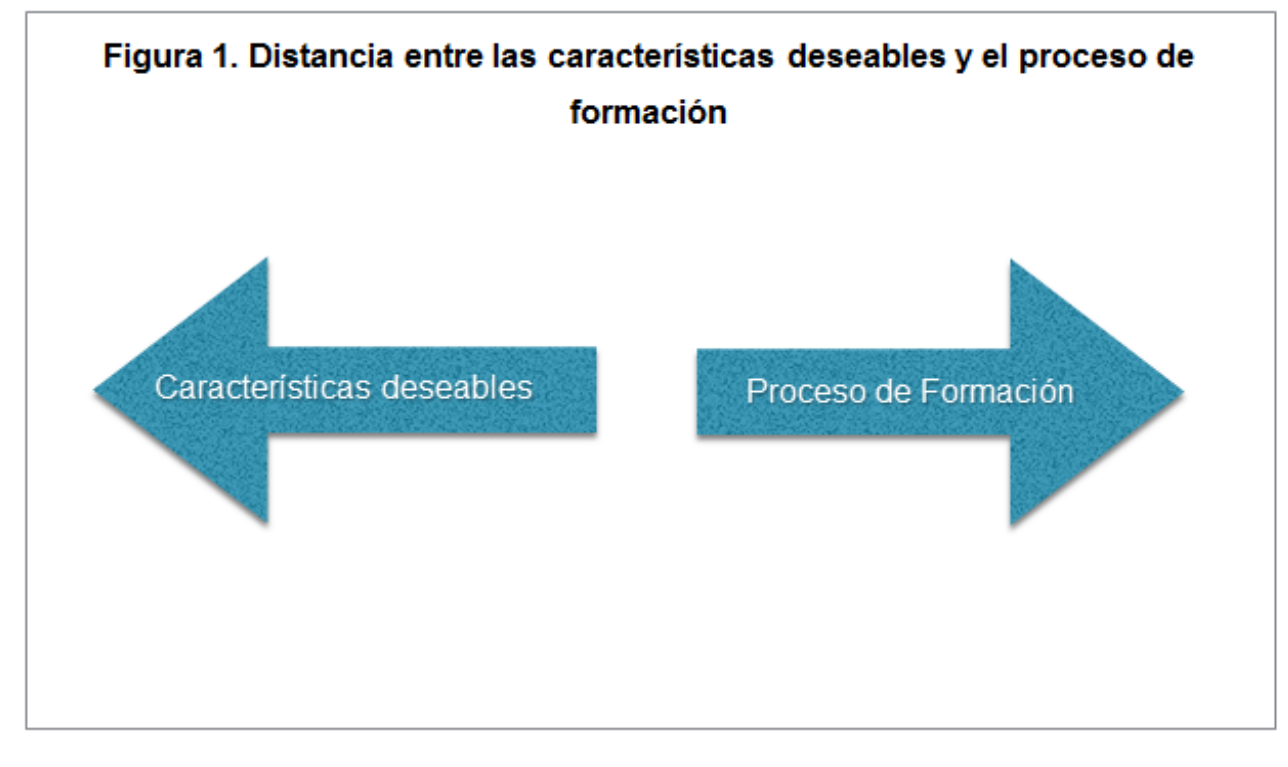

Fuente: Elaboración propia

De esta forma, conocer sobre las características deseables muestra "el piso" positivo para cualquier aprendizaje (Bertoni, 2005) e identifica qué tan próximas están dichas características al inicio del proceso de formación. Por tanto, se podría 
Reflexiones que promueven la calidad en los procesos de formación docente de la carrera de Educación Especial con Énfasis en Integración de la Universidad Nacional

Heidy León Arce, Ana Lucía Chaves Álvarez, Ana Isabel Herrera Castro, Sylvia Segura Esquivel, Ana María Hernández Segura y Lilliam Susana Ruiz Guevara

considerar que a mayor fortaleza de características deseables mejor pronóstico de desempeño en el proceso de formación.

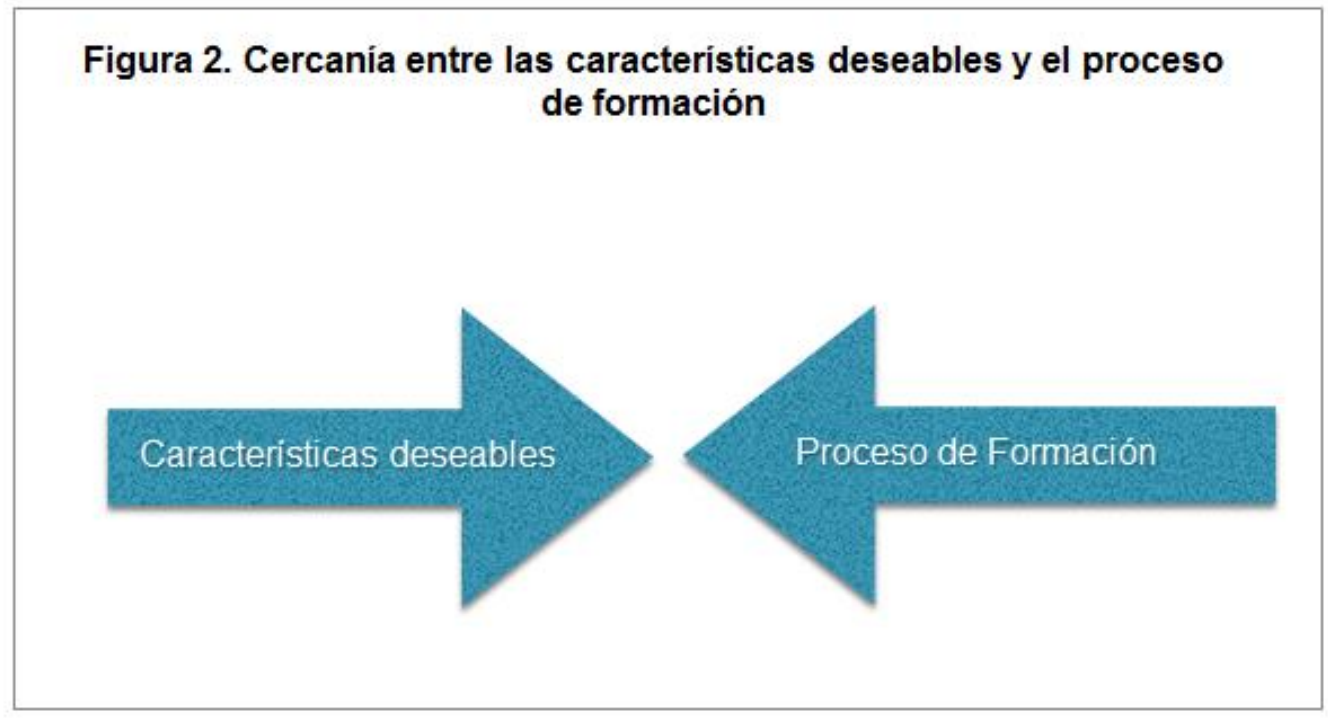

Fuente: Elaboración propia

Esto nos lleva a reflexionar sobre la importancia de tomar en cuenta en el desarrollo del plan de estudios características deseables como las disposiciones pedagógica incipiente, actitudinal, social y creativa, así como las habilidades lingüísticas, entre otras. Esto se convierte en una complementariedad entre los "intereses, expectativas, motivaciones y saberes por experiencia y no escolarizados" (Bertoni, p. 32, 2005) y la propuesta curricular de la CEEl.

Cabe señalar que la población estudiantil determina una priorización para las características, dejando en menor nivel de importancia, por ejemplo, las habilidades lingüísticas (fluidez verbal y escrita), la creatividad y el dinamismo. Algunos autores han manifestado que estas características son fundamentales, ya que cada vez se exigen más personas capaces de interpretar y transmitir órdenes, de entenderse con los compañeros para la complementación y las decisiones, y, fundamentalmente, con una sólida educación de base (expresión oral y escrita) y 
Reflexiones que promueven la calidad en los procesos de formación docente de la carrera de Educación Especial con Énfasis en Integración de la Universidad Nacional

Heidy León Arce, Ana Lucía Chaves Álvarez, Ana Isabel Herrera Castro, Sylvia Segura Esquivel, Ana María Hernández Segura y Lilliam Susana Ruiz Guevara

una clara capacidad de aprender rápido y constantemente nuevas prácticas (CEPAL, 1994, citado por Bertoni, 2005).

Además, Mauri, 2002, citado por Educar en la Diversidad (2005), proyecto de MERCOSUR, la OEA y la UNESCO, se refirió a que la formación de los y las docentes debe considerar los siguientes aspectos:

- Los docentes han de formarse tanto en el dominio de los recursos de información como en habilidades sociales, cognitivas y lingüísticas que les permitan adaptarse críticamente al cambio. La formación en valores éticos y morales es también fundamental para contribuir a la igualdad de oportunidades y el desarrollo de sociedades más justas.

- Capacidad de innovar para mejorar la práctica a través de procesos de reflexión e investigación de la propia acción. El aprendizaje de la práctica reflexiva requiere que las actividades de formación de los docentes consideren las características de los contextos en los que intervienen y los problemas reales que enfrentan en su práctica (p.6).

\section{Realidad y relación de la CEEI con el contexto}

El vínculo de la carrera con el contexto y la realidad es permeado tanto por el eje práctico pedagógico que atraviesa el currículo por medio de la naturaleza teóricopráctica de los cursos como por los procesos de intervención pedagógica, los métodos de investigación y la vinculación de proyectos propios de la carrera. En cada uno de estos dispositivos pedagógicos subyace una relación dialéctica (teoría-práctica). Indagar la opinión de los y las estudiantes sobre esto último, así como acerca de la relación de los cursos con situaciones de la realidad, es incursionar en cómo perciben ellos y ellas la vinculación con el contexto. La tabla 2 sintetiza las respuestas brindadas sobre la relación teoría-práctica y su relación con situaciones de la realidad. 
Reflexiones que promueven la calidad en los procesos de formación docente de la carrera de Educación Especial con Énfasis en Integración de la Universidad Nacional

Heidy León Arce, Ana Lucía Chaves Álvarez, Ana Isabel Herrera Castro, Sylvia Segura Esquivel, Ana María Hernández Segura y Lilliam Susana Ruiz Guevara

\section{Tabla 2. Opinión de la población estudiantil sobre la relación teoría-práctica y situaciones de la realidad desarrolladas en los cursos de la CEEI}

\begin{tabular}{|c|c|c|}
\hline Se integra la teoría y la práctica & & Porcentaje \\
\hline Casi nunca & & $1,69 \%$ \\
\hline A veces & & $6,78 \%$ \\
\hline Casi siempre & & $28,81 \%$ \\
\hline \multirow[t]{2}{*}{ Siempre } & & $62,71 \%$ \\
\hline & Total & $100,00 \%$ \\
\hline $\begin{array}{l}\text { Existe una estrecha relación con } \\
\text { situaciones de la realidad }\end{array}$ & & Porcentaje \\
\hline Casi nunca & & $3,39 \%$ \\
\hline A veces & & $6,78 \%$ \\
\hline Casi siempre & & $28,81 \%$ \\
\hline \multirow[t]{2}{*}{ Siempre } & & $61,02 \%$ \\
\hline & Total & $100,00 \%$ \\
\hline
\end{tabular}

Fuente: Instrumento estudiantes regulares proceso de autoevaluación de la CEEI 2010.

Chaves, Hernández, León, Ruiz y Segura (2010). Informe Paralelo: Cuestionario aplicado a Estudiantes Regulares de la carrera de Educación Especial. Proceso de Autoevaluación para la segunda Reacreditación. División de Educación Básica. Universidad Nacional (documento impreso, no publicado)

De acuerdo con la información anterior, un 91,52\% manifiesta que los cursos del plan de estudios de la CEEI siempre y casi siempre integran la teoría y la práctica en la cotidianidad, lo cual genera, como lo planteó Morin (2011), sentido, significado e interés para los y las estudiantes, y al mismo tiempo permite formar profesionales con capacidad para responder a esa realidad, ser propositivos en ella e impactar en los diferentes contextos en los que se desenvuelven para así buscar una mejora en la sociedad. 
Reflexiones que promueven la calidad en los procesos de formación docente de la carrera de Educación Especial con Énfasis en Integración de la Universidad Nacional

Heidy León Arce, Ana Lucía Chaves Álvarez, Ana Isabel Herrera Castro, Sylvia Segura Esquivel, Ana María Hernández Segura y Lilliam Susana Ruiz Guevara

Si bien la CEEI propicia la relación teoría-práctica y, a la vez, permite que el estudiantado conozca diferentes contextos, es necesario reflexionar sobre la importancia de seguir buscando y abriendo nuevos espacios emergentes.

Al respecto, un estudiante participante en el proceso de autoevaluación de la CEEI (Informe paralelo: Cuestionario aplicado a Estudiantes Regulares de la carrera de Educación Especial. 2010) planteó en el instrumento (en el apartado de "Comentarios") lo siguiente:

La educación especial necesita enfocarse a la diversidad, a los diversos contextos, ampliar un poco más. Me parece que las prácticas deberían de modificarse, realizarse en lugares en donde la universidad tenga convenios y en otros servicios diferentes que no sean escuelas, es importante educar a la población en general.

Así, otro estudiante agregó:

Pienso que sería importante en la carrera ofrecer oportunidades a los estudiantes de realizar la práctica en otros ámbitos y no solo en el escolar, ya que un profesional en educación de especial también debería participar en otros ámbitos, por ejemplo en inserción laboral.

Es importante señalar que los aportes de los y las estudiantes han permitido generar cambios en la ejecución del plan de estudios, fortaleciendo la postura conceptual para desarrollar criterio profesional y promover la práctica reflexivacrítica, el desarrollo de habilidades de gestión y negociación, el trabajo colaborativo, la contextualización y los conocimientos de realidades diversas. También esos insumos del estudiantado han propiciado la oportunidad de investigar y hacer prácticas tanto en contextos formales como en instituciones educativas del país que atienden poblaciones desde edades tempranas hasta 
Reflexiones que promueven la calidad en los procesos de formación docente de la carrera de Educación Especial con Énfasis en Integración de la Universidad Nacional

Heidy León Arce, Ana Lucía Chaves Álvarez, Ana Isabel Herrera Castro, Sylvia Segura Esquivel, Ana María Hernández Segura y Lilliam Susana Ruiz Guevara

edades adultas, por ejemplo las municipalidades, el Hospital Nacional de Niños, el Hospital Nacional Psiquiátrico, las asociaciones de personas con discapacidad, las organizaciones no gubernamentales, los servicios de apoyo educativo y el trabajo con otros grupos étnicos, entre otros. Esto revela que el trabajo que anteriormente se centraba en las aulas integradas y en la población con condición de discapacidad se abre hacia nuevas prácticas educativas y hacia nuevos apoyos que el entorno debe brindarles a las personas.

Por otra parte, resulta pertinente anotar que el plan de estudios de la CEEI no es únicamente un diseño curricular que está plasmado en un documento en específico, sino que, sin duda alguna, tiene que ver con el accionar, con la práctica, con la vivencia, con la experiencia y con la implementación de los aprendizajes construidos, aunado a otros aspectos fundamentales. Esta complementariedad entre teoría y práctica es lo que le da sentido a los procesos de formación superior. Precisamente, en cuanto a la importancia de la relación teoría-práctica Gimeno (2007) destacó lo siguiente.

El propio discurso sobre la relación teoría-práctica se nutre de la teoría y de las prácticas curriculares. Un discurso que debe desbordar los estrechos límites del aula. En la configuración y desarrollo del currículum podemos ver inter implicarse prácticas estrictamente didácticas; dentro de todas ellas juegan supuestos muy distintos, teorías, perspectivas e intereses muy diversos, aspiraciones y gestión de realidades existentes, utopía y realidad. La comprensión del currículum, la renovación de la práctica, la mejora de la calidad de la enseñanza a través del currículum no deben olvidar todas esas interrelaciones [en cursiva en el original] (p.33).

Es decir, la teoría y la práctica son elementos fundamentales del currículo, y es esto lo que procura la mejora en los procesos educativos, pues no se convierten en procesos aislados, sino en espacios de transformación, diálogo y reflexión a 
Reflexiones que promueven la calidad en los procesos de formación docente de la carrera de Educación Especial con Énfasis en Integración de la Universidad Nacional

Heidy León Arce, Ana Lucía Chaves Álvarez, Ana Isabel Herrera Castro, Sylvia Segura Esquivel, Ana María Hernández Segura y Lilliam Susana Ruiz Guevara

partir de la realidad en la que se encuentran inmersos. Lo que se busca en la CEEI es una estrecha relación entre ambos elementos para formar un docente crítico, reflexivo, capaz de desarrollar procesos de praxis, más que teoría o práctica. Al respecto, Freire (2004) planteó que:

La praxis no es la acción ciega, desprovista de intención o finalidad. Es acción y reflexión. Mujeres y hombres son seres humanos porque se han hecho históricamente seres de la praxis y así se han vuelto capaces de, transformando el mundo, conferirle significado. Es que, como seres de la praxis y solamente en cuanto tales, al asumir una situación concreta en la que estamos, como condición desafiante, somos capaces de modificar su significación por medio de nuestra acción (pp.28-29).

Los argumentos anteriores sirven de base para agregar que en el plan de estudios de la CEEI, de acuerdo con las opiniones de los y las estudiantes participantes del proceso de acreditación 2011, existe una estrecha relación entre el plan de estudios y las situaciones de la realidad. Esto se refleja en el hecho de que el $89,83 \%$ de ellos manifiesta que siempre y casi siempre esto ocurre (tabla 2 ). Se deduce entonces que esta relación con el contexto permite buscar soluciones a problemas sociales, actuar y tomar decisiones en los contextos específicos en los que los y las estudiantes se desenvolverán profesionalmente. Al mismo tiempo, este contacto con el contexto permite la formación de docentes investigadores, innovadores y reflexivos. Esto coincide con lo que Rodríguez y Castañeda (2001) señalaron:

Podría pensarse que el significado que tiene para los docentes poder participar en procesos de innovación y de investigación que tienen lugar en la cotidianidad escolar llena en gran medida los vacíos en la formación, pero, sobre todo, incita a la búsqueda autónoma de nuevos caminos pedagógicos $\mathrm{e}$ induce a un cambio en las mentalidades, en las representaciones y en los estereotipos que manejan los docentes de sí 
Reflexiones que promueven la calidad en los procesos de formación docente de la carrera de Educación Especial con Énfasis en Integración de la Universidad Nacional

Heidy León Arce, Ana Lucía Chaves Álvarez, Ana Isabel Herrera Castro, Sylvia Segura Esquivel, Ana María Hernández Segura y Lilliam Susana Ruiz Guevara

mismos, de su profesión y de la escuela. De igual manera, posibilita el desarrollo de la capacidad crítica, la autorreflexión y la interpretación de sus prácticas pedagógicas, la comprensión del mundo en que viven, todo esto como manera de creación de conciencia sobre sí mismos y sobre su trabajo docente (s.p).

De este modo, se pretende que el plan de estudios de la CEEl responda a las necesidades actuales de la sociedad y propicie una formación con sentido y con el propósito de transformar realidades para mejorar la sociedad. Quedan aún muchos retos y aspectos por mejorar, sin embargo, se considera que en la medida en la que los y las estudiantes sigan asumiendo un rol participativo y protagónico los procesos de formación superior irán creciendo y mejorando significativamente.

\section{Pertinencia del plan de estudios: investigación sobre temas afines a la carrera}

La pertinencia, entendida como la perspectiva que posee la carrera enmarcada en la misión y visión de una universidad estatal, debe responder a los objetivos que como institución se han planteado, en concordancia con las necesidades de la sociedad actual. De esta manera, tanto el eje de investigación de la carrera que atraviesa todos los cursos como el área de investigación donde se inscriben los cursos propios de esta área, así como los proyectos de la carrera, ofrecen espacios y dinámicas que propician intercambios, negociaciones, propuestas y ejecuciones de acciones que respondan a necesidades de ámbitos concretos, sean aulas, comunidades o poblaciones. La tabla 3 refleja la opinión de los y las estudiantes sobre si se llevan a cabo investigaciones afines a la carrera. 
Reflexiones que promueven la calidad en los procesos de formación docente de la carrera de Educación Especial con Énfasis en Integración de la Universidad Nacional

Heidy León Arce, Ana Lucía Chaves Álvarez, Ana Isabel Herrera Castro, Sylvia Segura Esquivel, Ana María Hernández Segura y Lilliam Susana Ruiz Guevara

\section{Tabla 3. Investigación en temas afines a la carrera}

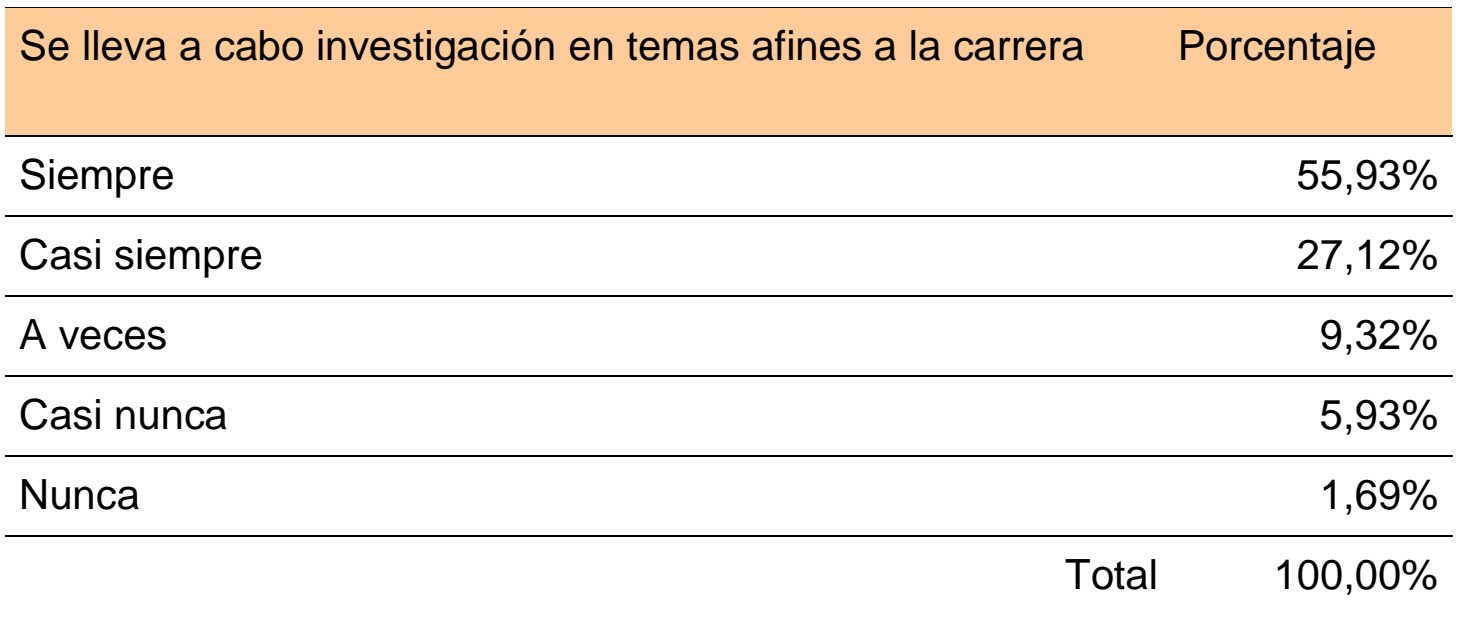

Fuente: Instrumento estudiantes regulares proceso de autoevaluación de la CEEI 2010.

Chaves, Hernández, León, Ruiz y Segura (2010). Informe Paralelo: Cuestionario aplicado a Estudiantes Regulares de la carrera de Educación Especial. Proceso de Autoevaluación para la segunda Reacreditación. División de Educación Básica. Universidad Nacional (documento impreso, no publicado)

En cuanto a la opinión de la población estudiantil participante en el proceso de acreditación de la CEEI 2011con relación a si se lleva a cabo investigación en temas afines a la carrera, el $83,05 \%$ afirma que siempre y casi siempre se realiza, lo que evidencia que la investigación es una fortaleza de su formación universitaria.

Es importante señalar que en el período comprendido entre el 2010-2013 se han desarrollado en la CEEl investigaciones en el marco del paradigma de investigación cualitativa con un enfoque de investigación-acción, que se amplía a diferentes contextos relacionados con la inserción laboral con personas con discapacidad; la promoción de la autonomía y la vida independiente; la salud ocupacional de los y las docentes de educación especial; la accesibilidad y atención a las necesidades educativas del Programa Educación Abierta; la educación inclusiva; la negociación y gestión con instituciones en las 
Reflexiones que promueven la calidad en los procesos de formación docente de la carrera de Educación Especial con Énfasis en Integración de la Universidad Nacional

Heidy León Arce, Ana Lucía Chaves Álvarez, Ana Isabel Herrera Castro, Sylvia Segura Esquivel, Ana María Hernández Segura y Lilliam Susana Ruiz Guevara

comunidades; y el trabajo con familias, entre otros contextos. Cabe destacar que estas líneas de investigación responden a las necesidades y los intereses del contexto, así como a las relaciones que tienen las y los estudiantes de la CEEI con sus procesos de investigación y práctica educativa.

Sin embargo, aunque esto revela un aspecto positivo de la CEEl, también es importante seguir trabajando en la reflexión con respecto al impacto de estas investigaciones en la realidad. En cuanto a lo anterior, dos estudiantes participantes en el proceso de acreditación de la CEEI 2010, señalaron que "en la licenciatura, al momento de realizar la tesis, se enfoque más en su implementación para mejorar la sociedad y no solamente un documento que se guarda en una biblioteca y llega a empolvarse" y "la posibilidad de realizar un trabajo práctico para la tesis, como por ejemplo trabajo de alfabetización" (Informe paralelo: Cuestionario aplicado a Estudiantes Regulares de la carrera de Educación Especial, 2010).

Precisamente estas respuestas evidencian que, si bien es cierto que hay logros y la CEEI impulsa la investigación como un eje fundamental de la carrera, aún quedan retos por asumir, de modo que estas investigaciones logren un mayor impacto y pertinencia en las realidades socioeducativas.

\section{Reflexiones finales}

A la luz del proceso de autoevaluación, del análisis de la información brindada por la población estudiantil y de las experiencias de la CEEI, se considera que son más los hallazgos que se identifican y los desafíos que quedan por ejecutar en la carrera, por lo que se exponen algunas reflexiones finales a continuación:

De acuerdo con la consulta realizada sobre las características deseables, la población estudiantil participante en el proceso de acreditación de la CEEI 2011 
Reflexiones que promueven la calidad en los procesos de formación docente de la carrera de Educación Especial con Énfasis en Integración de la Universidad Nacional

Heidy León Arce, Ana Lucía Chaves Álvarez, Ana Isabel Herrera Castro, Sylvia Segura Esquivel, Ana María Hernández Segura y Lilliam Susana Ruiz Guevara

las prioriza según sus marcos referenciales y experimentales; sin embargo, haremos una organización en tres bloques que permiten visualizar la importancia que la carrera le va a dar a posibles áreas de desarrollo de características deseables.

En el primer grupo los resultados demuestran que la población estudiantil prioriza la sensibilidad para trabajar con personas con discapacidad, característica que permite visualizar que los y las estudiantes han vivenciado acercamientos con grupos sociales, familiares, centros educativos, entre otros, con dicha población, y sus experiencias previas los llevan a considerar que es fundamental para poder ingresar a un proceso de formación de este tipo.

En el segundo grupo cabe señalar las habilidades para enseñar y las destrezas interpersonales, así como la creatividad y el dinamismo, que son reconocidas por la CEEI como elementos propios de la disciplina y ubicadas en un área pedagógica. En el tercer grupo la población estudiantil ubica las habilidades verbales y escritas en un grado de menor importancia; sin embargo, para la CEEI existe un dilema, debido a que se considera que dichas habilidades lingüísticas representan saberes conceptuales, procedimentales y actitudinales fundamentales en el desarrollo del proceso de formación que repercute en las prácticas pedagógicas.

La importancia de haber preguntado sobre las características deseables va más allá de una priorización de estas, pues se comprende la necesidad que posee la CEEI de identificar estas y otras características para valorar la distancia con la que ingresan a enfrentar un proceso formativo que requiere no solo habilidades básicas sino una mirada más compleja ante las realidades socioeducativas. La CEEI plantea características deseables no como un perfil o una condición, sino 
Reflexiones que promueven la calidad en los procesos de formación docente de la carrera de Educación Especial con Énfasis en Integración de la Universidad Nacional

Heidy León Arce, Ana Lucía Chaves Álvarez, Ana Isabel Herrera Castro, Sylvia Segura Esquivel, Ana María Hernández Segura y Lilliam Susana Ruiz Guevara

como un desafío que le permita a la carrera desarrollar acciones y estrategias que favorezcan los procesos de formación para así disminuir dicha brecha.

La línea de investigación-acción en la CEEI es una respuesta a las necesidades socioeducativas. La operacionalización de dicha línea se puede visualizar desde las prácticas pedagógicas de los cursos, o bien desde las propuestas de los trabajos finales de graduación que se aproximan a ámbitos no formales. Esto lleva a la CEEI a replantearse habilidades no consideradas en el plan de estudios, como la gestión y la negociación. Mirar los procesos pedagógicos no solo en el ámbito escolar, sino en espacios no formales es fundamental. Dichos espacios han llevado a la CEEI a desarrollar habilidades de gestión y negociación en los cursos.

La naturaleza teórico-práctica de los cursos, a través del eje práctico-pedagógico, promueve la vinculación y la identificación de las necesidades reales del contexto, lo que genera en el y la estudiante una postura crítica y reflexiva en su proceso de formación. Por ende, la relación teoría-práctica le permite tener al estudiantado una visión más amplia y pertinente de las necesidades y realidades socioeducativas, por lo que la CEEI debe asumirla como praxis.

Las áreas temáticas desarrolladas en los trabajos finales de graduación se evidencian en dos aspectos. Por un lado, la línea de investigación-acción vislumbra nuevos espacios emergentes que van desde el ámbito escolar y fuera de él, desde un cambio de mirada metodológica más diversa, amplia y humana que promueve las interacciones, la convivencia y la proximidad con el contexto real y lo cotidiano. Y por el otro, los procesos de autoevaluación permiten la participación de los diferentes actores y una reflexión al interior de la carrera que conlleva a una mejora de los procesos educativos universitarios. 
Reflexiones que promueven la calidad en los procesos de formación docente de la carrera de Educación Especial con Énfasis en Integración de la Universidad Nacional

Heidy León Arce, Ana Lucía Chaves Álvarez, Ana Isabel Herrera Castro, Sylvia Segura Esquivel, Ana María Hernández Segura y Lilliam Susana Ruiz Guevara

Los y las estudiantes de la CEEI manifiestan que el proceso de formación es pertinente, razón por la cual se podría decir que les permite trabajar y desenvolverse con poblaciones diversas, siendo sensibles y críticos ante la realidad en la que se encuentran inmersos.

En relación con la búsqueda de soluciones a problemas sociales, y su vinculación con los diversos contextos, la carrera le permite desarrollar al estudiantado la capacidad de adaptarse a nuevas situaciones, la toma de decisiones, la disposición y el interés por trabajar con diversas poblaciones, así como el trabajo en equipo. Es decir que se requiere un y una estudiante que puedan leer la realidad y transformarla para impactar en dicho contexto.

\section{Referencias}

Bertoni, E. (2005). El estudiante universitario: una aproximación al perfil de ingreso. Comisión Sectorial de Enseñanza Unidad Académica. "Articulación y Flexibilización Curricular en las carreras técnicas, tecnológicas y de grado de la Universidad de la República" Recuperado de http://www.cse.edu.uy/sites/www.cse.edu.uy/files/documentos/EL\%20ESTU DIANTE\%20UNIVERSITARIO.pdf

Chaves, A.L. Hernández. A.M., León, H, Ruiz. S., Y Segura, S. (2010). Informe Paralelo Cuestionario aplicado a Estudiantes Regulares de la carrera de Educación Especial. Proceso de Autoevaluación para la segunda Reacreditación. División de Educación Básica. Universidad Nacional (documento impreso, no publicado)

Comisión de Expertos de Educación Especial. (2004). Nuevas perspectivas y visión de la educación especial. Informe de la Comisión de expertos. Ministerio de Educación, Gobierno de Chile. Recuperado de http://www.rmm.cl/usuarios/equiposite/doc/200603021238250.informe_expe rtos_educ_especial.pdf

Educar en la Diversidad. (2005). Material de Formación Docente. MERCOSUR, OEA $y$ UNESCO. Recuperado de http://www.inclusioneducativa.org/content/documents/educar_diversidad.pdf 
Reflexiones que promueven la calidad en los procesos de formación docente de la carrera de Educación Especial con Énfasis en Integración de la Universidad Nacional

Heidy León Arce, Ana Lucía Chaves Álvarez, Ana Isabel Herrera Castro, Sylvia Segura Esquivel, Ana María Hernández Segura y Lilliam Susana Ruiz Guevara

Freire, P. (2004). La importancia de leer y el proceso de liberación. México: Siglo veintiuno editores.

Freire, P. (2009). Pedagogía de la autonomía. Saberes necesarios para la práctica educativa. México: Siglo veintiuno editores.

Gimeno, J. (2007). El currículum: una reflexión sobre la práctica (9. ${ }^{a}$ ed.). Madrid, España: Ediciones Morata.

Lima, D., Kahan,S., García, R., Ríos, C., Cha, A. y Plachot, G. (2004). Características deseables de la formación del estudiante al ingreso a la Universidad. Recuperado de http://www.fing.edu.uy/ skahan/completo.pdf

Lucha, R. y Baerlocher, C. (2008). Las habilidades interpersonales de un profesor de e/le en prácticas: estudio de caso. XIX Congreso Internacional de la ASELEEl profesor de español LE/L2. Cáceres, 2008. pp. 583-593.Centro virtual Cervantes. Recuperado en http://cvc.cervantes.es/ensenanza/biblioteca_ele/asele/asele_xix.htm

Martínez-Salanova , E. (2002 ). Propuestas críticas y creativas para vivir en la nueva sociedad mediática. Revista Ágora digital, 3, 1-23. Primer semestre 2002.

Morin, E. (2011). Los siete saberes necesarios para la educación del futuro. Barcelona, España: Paidós.

Ordóñez, J. (2010). Vida, pensamiento y obra de Paulo Freire. Revista Umbral, 2(7), 43-48.

Pereira. Z. (2010). Las dinámicas interactivas en el ámbito universitario: el clima de aula. Revista Educare,14, vol. XIV, pp. 7-20 (número extraordinario). Recuperado de http://revistas.una.ac.cr/index.php/EDUCARE/article/viewFile/1524/444

Rodríguez, J. G. y Castañeda, E. (2001). Los profesores en contextos de investigación e innovación. Revista Iberoamericana de Educación, 25, 103146. Recuperado de http://www.rieoei.org/rie25a05.htm 
Reflexiones que promueven la calidad en los procesos de formación docente de la carrera de Educación Especial con Énfasis en Integración de la Universidad Nacional

Heidy León Arce, Ana Lucía Chaves Álvarez, Ana Isabel Herrera Castro, Sylvia Segura Esquivel, Ana María Hernández Segura y Lilliam Susana Ruiz Guevara

Sartori, M.L., y Castilla, M.E. (Comp. 2004). Educar en la diversidad ¿realidad o utopía?. Editorial ffha-UNSJ, San Juan, Argentina

Sverdick, I., Anderson, G.,Augustowsky, G., Herr, K., Rivas, I. y Suárez, D. (2007). La investigación educativa. Una herramienta de conocimiento y de acción. Buenos Aires, Argentina: Centro de Publicaciones Educativas y Material Didáctico.

Tedesco, J. y Tenti Fanfani, E. (2002). Nuevos tiempos y nuevos docentes. Conferencia Regional: El desempeño de los maestros en América Latina y el Caribe: Nuevas prioridades, Brasil. Recuperado de http://unesdoc.unesco.org/images/0013/001346/134675so.pdf

Toro, J. M. (2005). Educar con «Co-razón» (6. ${ }^{\text {aed. }}$. España: Desclée De Brouwer.

Tünnermann, C. (2009). Pertinencia y calidad de la Educación Superior. Ponencia presentada en el Seminario Tendencias y Desafíos en la Educación Superior, en el marco de la Conferencia Mundial de la Educación Superior, París, Francia.

Universidad Nacional (2005). Modelo pedagógico. Heredia, Costa Rica.

Universidad Nacional (2008). Plan de estudios de la carrera de Educación Especial con Énfasis en Integración. División de Educación Básica, CIDE. Heredia, Costa Rica.

Zúñiga, M. (2010). Relevancia de la sensibilidad pedagógica ante la Diversidad Educativa. Ponencia presentada en el Congreso Iberoamericano de Educación, Metas 2021, Argentina. Recuperado de http://www.chubut.edu.ar/descargas/secundaria/congreso/EDUCINCLUSIVA /RLE2370_Zuniga.pdf 